\title{
Lymphatic invasion predicts survival in patients with early node-negative non-small cell lung cancer
}

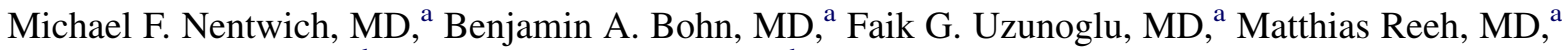 \\ Alexander Quaas, MD, ${ }^{\mathrm{b}}$ Tobias J. Grob, MD, PhD, ${ }^{\mathrm{b}}$ Daniel Perez, MD, ${ }^{\mathrm{a}}$ Asad Kutup, MD, ${ }^{\mathrm{a}}$ \\ Maximilian Bockhorn, MD, ${ }^{\mathrm{a}}$ Jakob R. Izbicki, MD, FACS, FRCS, ${ }^{\mathrm{a}}$ and Yogesh K. Vashist, MD ${ }^{\mathrm{a}}$
}

Objective: The aim of this study was to assess the influence of lymphatic and vascular invasion on overall survival in patients with surgically resected non-small cell lung cancer (NSCLC) without lymph node and distant metastases.

\begin{abstract}
Methods: From January 1999 to December 2009, a total of 190 NSCLC patients with node-negative pT1-pT4 disease underwent radical resection with lymphadenectomy. Pathologic reports were reclassified to the TNM-7 version, and the influence of lymphatic and vascular invasion on overall survival was examined using Kaplan-Meier and adjusted Cox proportional hazards analyses.
\end{abstract}

\begin{abstract}
Results: Lymphatic invasion was present in 34 (17.9\%) and vascular invasion in 28 (14.7\%) of 190 cases. Lymphatic and vascular invasions were correlated with higher Union for International Cancer Control stages ( $P=.056$ and $P=.011$, respectively) and poor differentiated tumors $(P=.051$ and $P=.012$, respectively). There was no difference between pT1a and pT1b tumors in the presence of lymphatic $(P=.912)$ or vascular $(P=.134)$ invasion. Survival analyses revealed lymphatic $(P<.001)$ and vascular $(P=.008)$ invasion as statistically significant for the entire study population. Multivariable Cox analysis adjusted for age, Union for International Cancer Control stage, and lymphatic and vascular invasion confirmed lymphatic, but not vascular, invasion as an independent prognostic factor $(P<.001$; hazard ratio, 3.002; 95\% confidence interval, 1.780-5.061). Especially in early stages, lymphatic invasion was associated with poorer overall survival in pT1a $(P<.001)$, pT1b $(P=.019)$, and pT2a $(P=.028)$ tumors.
\end{abstract}

Conclusions: Lymphatic invasion represents an independent risk factor for node-negative NSCLC. Its implications on therapy decision making should be further evaluated, especially in early stages. (J Thorac Cardiovasc Surg 2013;146:781-7)

Even in the early stages of non-small cell lung cancer (NSCLC), patients form a heterogeneous group with interindividual differences in survival. To improve the staging, the seventh edition of tumor, nodes, and metastasis (TNM) classification for NSCLC was introduced in 2009. ${ }^{1}$ Yet, nonanatomic cancer characteristics, such as tumor grading and vascular and lymphatic invasion, have not been considered as influencing factors on stage classifications. ${ }^{2}$

To apply individual therapeutic concepts based on individual tumor biology, additional information routinely available

\footnotetext{
From the Department of General, Visceral and Thoracic Surgery ${ }^{\mathrm{a}}$ and the Institute of Pathology, ${ }^{\mathrm{b}}$ University Medical Center Hamburg-Eppendorf, Hamburg, Germany. Disclosures: T.J.G. reports lecture fees from Astra Zeneca, Roche Pharma, and Abbott Molecular. The other authors have nothing to disclose with regard to commercial support.

M.F.N. and B.A.B. contributed equally to this work and, therefore, share first authorship.

Presented at the 21st Congress of the German Society for Thoracic Surgery, Karksruhe, Germany, September 27-29, 2012.

Received for publication Sept 30, 2012; revisions received April 14, 2013; accepted for publication April 24, 2013; available ahead of print June 17, 2013.

Address for reprints: Yogesh K. Vashist, MD, Department of General, Visceral and

Thoracic Surgery, University Medical Center Hamburg-Eppendorf, Martinistrasse

52, 20246 Hamburg, Germany (E-mail: vashist@uke.de).

$0022-5223 / \$ 36.00$

Copyright $(2013$ by The American Association for Thoracic Surgery

http://dx.doi.org/10.1016/j.jtcvs.2013.04.037
}

in histopathologic specimen evaluation may add to improve the assessment of a tumor risk profile and help to stratify patients in need of an adjuvant therapy. The group of patients with stage IA NSCLC is especially of interest, because adjuvant therapy is generally not administrated in this setting.

In other solid malignancies, such as breast, colon, and gastric cancer, lymphatic invasion has already been identified as a negative prognostic factor for survival. ${ }^{3-5}$ In NSCLC, the influence of lymphatic and vascular invasion on patient prognosis and its role in therapy planning are still under debate.

Therefore, the aim of the present study was to evaluate the role of lymphatic and vascular invasion as a potential factor influencing patient survival in our study population, especially in the early stages.

\footnotetext{
METHODS

Patients

We retrospectively reviewed the records of 190 patients with NSCLC (pT1-pT4 N0M0), with available information on lymphatic and vascular invasion status, who underwent surgical resection at the Department of General, Visceral and Thoracic Surgery, University Medical Center Hamburg-Eppendorf (Hamburg, Germany) between January 1999 and December 2009.
} 


\section{Abbreviations and Acronyms \\ $\mathrm{CT}=$ computed tomographic \\ NSCLC $=$ non-small cell lung cancer \\ TNM = tumor, nodes, and metastasis \\ UICC = Union for International Cancer Control \\ YSR = year survival rate}

Preoperative evaluation included patients' medical history, physical examination, plain chest X-ray, thoracic computed tomographic (CT) scan, bone scan, abdominal ultrasound, and fiberoptic bronchoscopy and positron emission tomography-CT, according to national guidelines. ${ }^{6}$ Mediastinoscopy, brain magnetic resonance imaging, or CT scan was only performed in selected cases.

Surgical procedures consisted of standard lobectomy, bilobectomy, and pneumonectomy. All resections included a systematic dissection of hilar and mediastinal lymph node stations, according to the Japan Lung Cancer Society map. ${ }^{7}$ Data collection was done prospectively. Follow-up was done on a regular outpatient setting via contacting the patient or the patient's primary care physician and included data on deaths of any cause. The study was approved by the Ethics Committee of the Chamber of Physicians in Hamburg, and written informed consent was obtained from all patients participating in this study.

Patients were excluded from this study if a synchronous or metachronous (up to 1 year after first resection) second malignancy other than NSCLC was present, in cases with tumor residual disease (R1 or R2) or if a wedge resection without systematic lymphadenectomy was performed. Patients with 30-day-mortality were also excluded to avoid blurring of survival estimation, as were patients with a follow-up shorter than 6 months (Figure 1). The mean follow-up of the censored patients was 55.2 months (range, 8.6-197.0 months).

The mean patient age was 65.1 years (range, 38.1-91.9 years). Surgical procedures included $153(80.0 \%)$ lobectomies, $12(6.3 \%)$ bilobectomies, 11 atypical resections with lymphadenectomy (5.8\%), and $14(7.4 \%)$ pneumonectomies. Lymphatic invasion was present in $34(17.9 \%)$, and vascular invasion was present in $28(14.7 \%)$, of 190 cases.

\section{Histopathologic Evaluation}

Histopathologic studies included characteristics on tumor histology (adenocarcinoma, squamous cell carcinoma, and large-cell carcinoma), tumor location, tumor size, tumor grading, lymph node status, lymphatic invasion, vascular invasion, and resection margin status. To assess the lymphatic and vascular invasion status, sections were stained routinely by the Elastica van Gieson and the hematoxylin-eosin methods. Histopathologic diagnosis of lymphangioinvasion was made by a board-certified pathologist on clearly distinguishable clusters of tumor cells ( $\geq 4$ tumor cells) within spaces with endothelial lining in close relationship with venous vessels. In all cases with unclear findings, additional immunohistochemical staining using antibodies specific for endothelial cells (CD31 and D2-40; Dako, Glostrup, Denmark) was performed. The evaluation also included cytokeratin staining for verification of carcinoma cells within lymphatic vessels. Exemplarily histopathologic sections are given in Figure 2. All cases were classified or reclassified based on the seventh edition of the TNM classification system. ${ }^{1}$

\section{Statistical Analysis}

Continuous data are given as median, including ranges. Categorical variables are shown as numbers and percentages. Long-term survival was estimated using the nonparametric product limit method (Kaplan-Meier). Multivariate Cox proportional hazards regression models were used for examination of competing risk factors (including parameters with univariate $P<.10$ ) and given with corresponding hazard ratios and $95 \%$ confidence intervals. Statistical significance was assumed at 2-tailed $P<.05$. Statistical calculation was performed with the PASW 18.0 Software package (SPSS Inc, Chicago, Ill), and survival curves were plotted with the R statistics software 2.15.2 (R Foundation for Statistical Computing, Vienna, Austria).

\section{RESULTS}

Table 1 depicts the tumor-specific characteristics of the entire study population, stratified according to the presence of lymphatic and vascular invasion.

We showed a borderline significant correlation between lymphatic $(P=.056)$ and a significant correlation between vascular $(P=.008)$ invasion and Union for International Cancer Control (UICC) stages. In addition, a parallel association between presence of lymphatic and vascular invasion was evident $(P<.001)$. Lymphatic $(P=.051)$ and vascular $(P=.012)$ invasions were more often present in poorer differentiated tumors. Interestingly, there was no difference between pT1a and pT1b tumors in the presence of lymphatic $(P=.912)$ or vascular $(P=.134)$ invasion.

\section{Impact of Lymphatic and Vascular Invasion on Survival}

Univariable analysis of the final study population revealed lymphatic invasion $(P<.001)$, vascular invasion $(P=.008)$ (Figure $3, A$ and $B)$, pT $(P<.001)$, and UICC stage $(P<.001)$ as statistically significant prognostic factors.

In multivariable Cox analysis, lymphatic invasion was confirmed as an independent prognostic factor $(P<.001$; hazard ratio, 3.002; 95\% confidence interval, 1.7805.061) for overall survival. All results of the univariable and multivariable analyses (including the parameters of lymphatic and vascular invasions, UICC stage, and age) are summarized in Table 2.

Applying the UICC staging system, the presence of lymphatic invasion negatively affected patient survival in stages IA $(P<.001)$ and IB $(P=.028)$, but not in higher stages. The 5-year survival rate (YSR) in stage IA patients was limited to $15 \%$ with the presence of lymphatic invasion in contrast to $68 \%$ without it. In stage IB, the 5 -YSR was $52 \%$ for patients without lymphatic invasion versus $31 \%$ for patients with lymphatic invasion (Figure 3, $C$ and $D$ ).

Because the UICC stage IA was composed of 43 pT1a and $39 \mathrm{pT} 1 \mathrm{~b}$ tumors, the influence of lymphatic invasion was assessed for each of the subgroups separately. Interestingly, lymphatic invasion was associated with poorer overall survival in pT1a $(P<.001)$ and pT1b $(P=.019)$ tumors. In addition, pT2a $(P=.028)$ tumors with positive lymphatic invasion had a poorer outcome, but not pT2b $(P=.085)$, pT3 $(P=.923)$, and pT4 $(P=.157)$ tumors.

The 5-YSR in pT1a patients was $14 \%$ with presence of lymphatic invasion compared with $75 \%$ without presence 


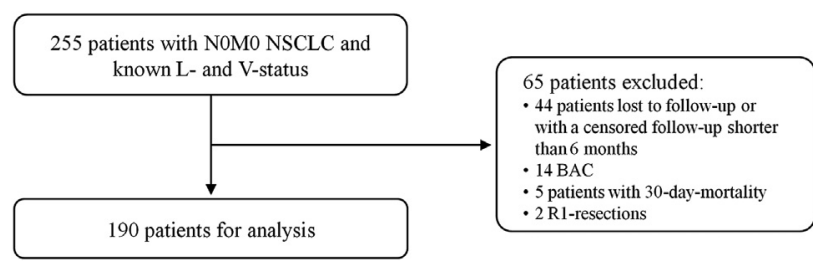

FIGURE 1. Flow diagram demonstrating the exclusion of patients. $L$, Lymphatic; V, vascular; NSCLC, non-small cell lung cancer; $B A C$, bronchioloalveolar carcinoma.

of lymphatic invasion $(P<.001)$. In pT1b patients, a similar survival trend was observed because the 5-YSR decreased to $16 \%$ if histologically the presence of lymphatic invasion was confirmed, whereas it was $61 \%$ without tumor cells penetrating lymphatic vessels $(P=.019)$.

Regarding vascular invasion, the 5-YSR in stage IA was $30 \%$ for patients with vascular invasion and $61 \%$ for patients without vascular invasion, without statistical significance $(P=.107)$ and possibly because of the few $\mathrm{V}+$ patients.

\section{DISCUSSION}

As our data show, the presence of lymphatic and vascular invasion is associated with a poorer patient outcome in early-stage NSCLC. Lymphatic invasion was identified as an independent risk factor in stage IA tumors. Interestingly, the negative effect of lymphatic and vascular invasion vanished in higher disease stages.
The process of metastasis formation is based on the ability of tumor cells to loosen from their primary tumor cell mass and invade to lymphatic and/or vascular structures. Therefore, the presence of angioinvasion demonstrates a state of already advanced and more aggressive tumor behavior. ${ }^{8}$ Because selected patients have a significantly reduced survival, although being treated at the same early stage of disease, additional factors not considered in the TNM classification influence the course of disease. ${ }^{9}$

Several studies report on a potential influence of lymphatic and vascular invasion on survival. ${ }^{10-12}$ These studies show a high discordance in design, volume, results, and conclusions. Early studies from Shields ${ }^{11}$ and Brechot and colleagues ${ }^{10}$ demonstrated a correlation between positive lymphatic invasion and poor prognosis for patients with NSCLC. However, Shields ${ }^{11}$ reported on no prognostic value for vascular invasion. In contrast, Goldstein and colleagues ${ }^{12}$ proposed lymphatic invasion as a prognostic factor for recurrence in their series of 218 stage I NSCLC cases. Performing separate analyses of lymphatic and vascular invasion and focusing on early disease stage, as done herein, 2 studies have reported on similar results. ${ }^{13,14}$ Hanagiri and colleagues ${ }^{13}$ showed, in 226 resected stage I NSCLC patients, a significantly poorer outcome for patients with positive lymphatic or positive vascular invasion. In a similar study with 229 resected stage I NSCLC patients, Funai and colleagues ${ }^{14}$ report on significantly worse survival of patients with lymphatic invasion compared with patients without lymphatic invasion. Contrarily, a study by Gabor and colleagues ${ }^{15}$ identified only

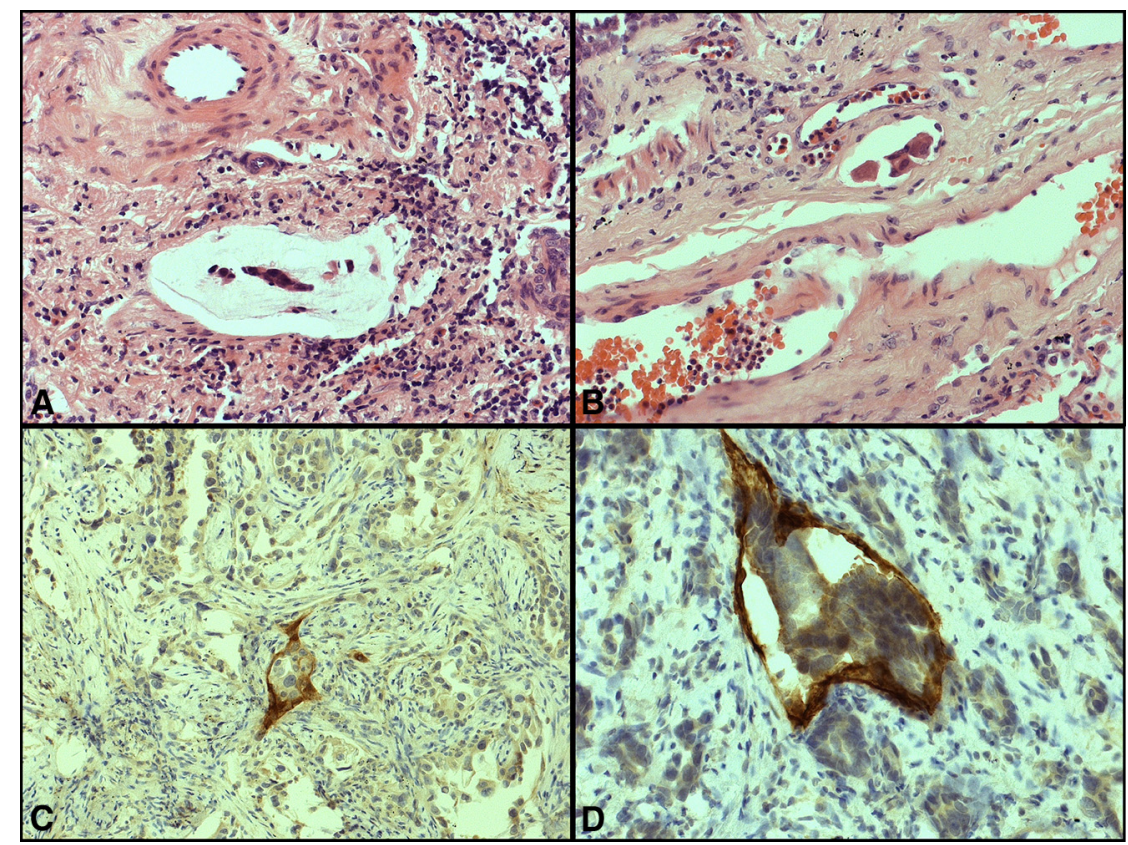

FIGURE 2. Representative tissue sections of non-small cell lung cancer tumors with the presence of lymphatic invasion stained by the hematoxylin-eosin methods (A and B) and with the monoclonal antibody D2-40 (C and D). 
TABLE 1. Lymphatic and vascular invasion in correlation to clinicopathologic data $(\mathbf{n}=190)$

\begin{tabular}{|c|c|c|c|c|c|c|}
\hline \multirow[b]{2}{*}{ Clinicopathologic features } & \multicolumn{3}{|c|}{ Lymphatic invasion } & \multicolumn{3}{|c|}{ Vascular invasion } \\
\hline & $\begin{array}{c}\text { Lo } \\
156(82.1) \\
\end{array}$ & $\begin{array}{c}\text { L1 } \\
34(17.9) \\
\end{array}$ & $P$ value & $\begin{array}{c}V 0 \\
162(85.3) \\
\end{array}$ & $\begin{array}{c}\text { V1 } \\
28(14.7) \\
\end{array}$ & $P$ value \\
\hline \multicolumn{7}{|l|}{ Age, y } \\
\hline$<60(\mathrm{n}=55)$ & $44(80.0)$ & $11(20.0)$ & & $47(85.5)$ & $8(14.5)$ & \\
\hline$\geq 60-<70(\mathrm{n}=66)$ & $52(78.8)$ & $14(21.2)$ & & $54(81.8)$ & $12(18.2)$ & \\
\hline$\geq 70(\mathrm{n}=69)$ & $60(87.0)$ & $9(13.0)$ & .414 & $61(88.4)$ & $8(11.6)$ & .558 \\
\hline \multicolumn{7}{|l|}{ Sex } \\
\hline Female $(n=61)$ & $55(90.2)$ & $6(9.8)$ & & $56(91.8)$ & $5(8.2)$ & \\
\hline Male $(\mathrm{n}=129)$ & $101(78.3)$ & $28(21.7)$ & .046 & $106(82.2)$ & $23(17.8)$ & .080 \\
\hline \multicolumn{7}{|l|}{ Tumor } \\
\hline pT1a $(n=43)$ & $36(83.7)$ & $7(16.3)$ & & $42(97.7)$ & $1(2.3)$ & \\
\hline pT1b $(n=39)$ & $33(84.6)$ & $6(15.4)$ & & $35(89.7)$ & $4(10.3)$ & \\
\hline pT2a $(n=63)$ & $55(87.3)$ & $8(12.7)$ & & $51(81.0)$ & $12(19.0)$ & \\
\hline $\mathrm{pT} 2 \mathrm{~b}(\mathrm{n}=21)$ & $17(81.0)$ & $4(19.0)$ & & $17(81.0)$ & $4(19.0)$ & \\
\hline pT3 $(\mathrm{n}=21)$ & $14(66.7)$ & $7(33.3)$ & & $16(76.2)$ & $5(23.8)$ & \\
\hline pT4 $(\mathrm{n}=3)$ & $1(33.3)$ & $3(66.7)$ & .085 & $1(33.3)$ & $2(66.7)$ & .011 \\
\hline \multicolumn{7}{|l|}{ UICC stage } \\
\hline IA $(n=82)$ & $69(84.1)$ & $13(15.9)$ & & 77 (93.9) & $5(6.1)$ & \\
\hline IB $(n=63)$ & $55(87.3)$ & $8(12.7)$ & & $51(81.0)$ & $12(19.0)$ & \\
\hline IIA $(n=20)$ & $16(80.0)$ & $4(20.0)$ & & $16(80.0)$ & $4(20.0)$ & \\
\hline $\mathrm{IIB}(\mathrm{n}=22)$ & $15(68.2)$ & $7(31.8)$ & & $17(77.3)$ & $5(22.7)$ & \\
\hline IIIA $(n=3)$ & $1(33.3)$ & $2(66.7)$ & .056 & $1(33.3)$ & $2(66.7)$ & .008 \\
\hline \multicolumn{7}{|l|}{ Histology } \\
\hline $\operatorname{ACC}(n=93)$ & $78(83.9)$ & $15(16.1)$ & & $83(89.2)$ & $10(10.8)$ & \\
\hline $\operatorname{SCC}(\mathrm{n}=74)$ & $63(85.1)$ & $11(14.9)$ & & $62(83.8)$ & $12(16.2)$ & \\
\hline $\operatorname{LCC}(n=23)$ & $15(65.2)$ & $8(34.8)$ & .077 & $17(73.9)$ & $6(26.1)$ & .160 \\
\hline \multicolumn{7}{|l|}{ WHO } \\
\hline $\mathrm{G} 1+\mathrm{G} 2(\mathrm{n}=111)$ & $96(86.5)$ & $15(13.5)$ & & $101(91.0)$ & $10(9.0)$ & \\
\hline $\mathrm{G} 3+\mathrm{G} 4(\mathrm{n}=77)$ & $58(75.3)$ & $19(24.7)$ & .051 & $60(77.9)$ & $17(22.1)$ & .012 \\
\hline
\end{tabular}

Data are given as number (percentage). $P$ values are calculated by cross-table analysis ( $\chi^{2}$ test). $A C C$, Adenocarcinoma; $L C C$, large-cell carcinoma; $S C C$, squamous cell carcinoma; UICC, Union for International Cancer Control; WHO, World Health Organization.

vascular, but not lymphatic, invasion in T1-3 N0 M0 NSCLC patients as adversely affecting survival.

Our series demonstrates a better outcome for pT1-pT4 N0 M0 patients without lymphatic and vascular invasion. The authors of 2 previously published large studies ( $\mathrm{n}=746$ and $\mathrm{n}=995)$ did not differ between lymphatic and vascular invasion. Ruffini and colleagues ${ }^{16}$ showed the negative effect of microscopic vascular invasion in pT1-T2 N0 NSCLC patients. Microscopic vessel invasion, found in $34 \%$ of all cases, was associated with poorer patient survival. ${ }^{16}$ Tsuchiya and colleagues ${ }^{17}$ suggested considering vessel invasion to the TNM staging system based on their studies of 995 patients with stages I and II disease.

Regarding the data presented herein, only lymphatic, not vascular, invasion significantly discriminated survival in the early stages. Possibly, the missing significance for vascular invasion results from low patient numbers in our study. Pechet and colleagues ${ }^{18}$ could demonstrate a drastically reduced survival in patients with positive vascular invasion in early NSCLC. Generally, separate analysis of both factors should be performed. In a recently published metastudy by Wang and colleagues, ${ }^{19}$ considering a total of
16,535 patients from 52 studies, blood vessel invasion occurred in a total of $29.8 \%$ and negatively affected overall survival in the overall patient cohort and especially in stage IA patients. In addition, the presence of both lymphatic and vascular invasion increased with increasing tumor stages in our study group. This might reflect a combination of a higher stochastical chance to invade vessels in larger tumors, and the increasing malignant potential of the tumor cells. As in higher stages, at least in our study population, the effect of vessel invasion did not influence survival anymore. It seems that vessel invasion is a distractor of malignant potential, especially in early NSCLC.

These findings support the hypothesis that the group of stage IA NSCLC patients is heterogeneous regarding their tumor risk profile, and a subgroup of these patients might benefit from adjuvant therapy. Tsuchiya and colleagues ${ }^{20}$ compared the survival rates of stage IA patients (staged by the fifth TNM classification system) receiving uraciltegafur therapy with and without vascular invasion. In their series, patients with vascular invasion had an improved survival with adjuvant therapy. ${ }^{20}$ Although large pooled metaanalyses failed to demonstrate a clear benefit of adjuvant 


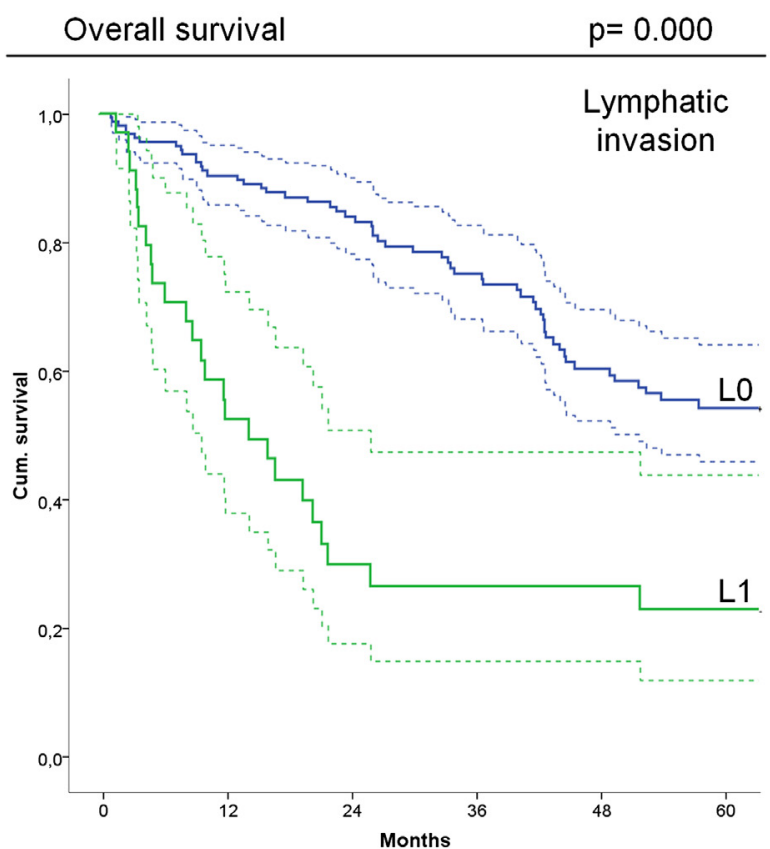

Numbers at risk (0-5 years)

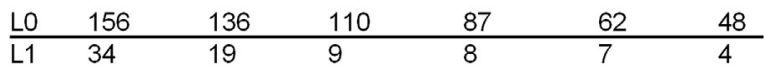

A

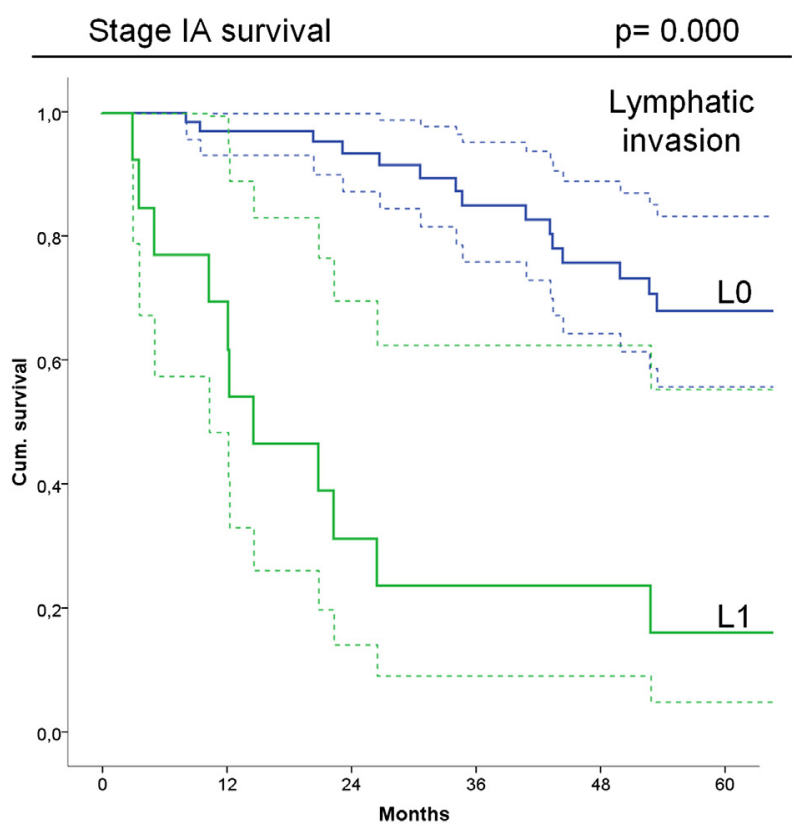

Numbers at risk (0-5 years)

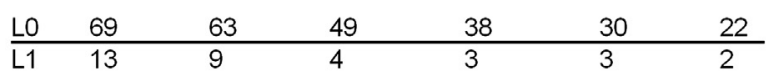

C

FIGURE 3. Estimated overall survival according to lymphatic invasion $(P=.000)(\mathrm{A})$, vascular invasion $(P=.008)(\mathrm{B})$, lymphatic invasion for Union for International Cancer Control (UICC) stage IA tumors $(P<.000)(\mathrm{C})$, and lymphatic invasion for UICC stage IB tumors $(P=.028)(\mathrm{D})(\mathrm{log}-\mathrm{rank}$ test, including $95 \%$ confidence intervals).

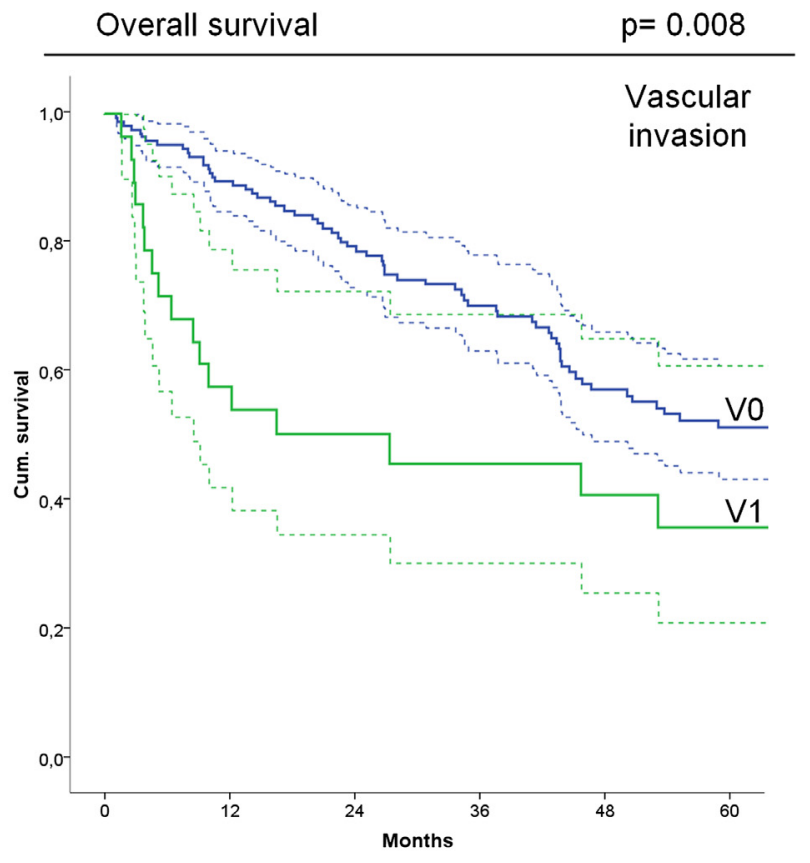

Numbers at risk ( $0-5$ years)

\begin{tabular}{lllllll}
$\mathrm{V} O$ & 162 & 139 & 108 & 85 & 61 & 46 \\
\hline $\mathrm{V} 1$ & 28 & 16 & 11 & 10 & 8 & 4
\end{tabular}

B

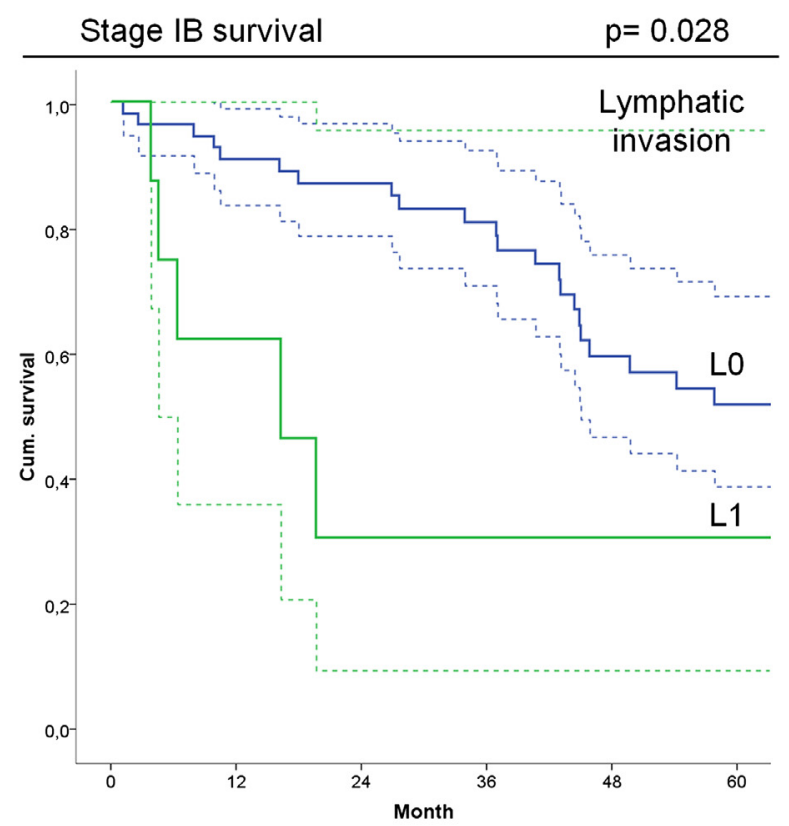

Numbers at risk ( $0-5$ years)

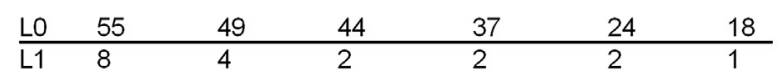

\section{D}


TABLE 2. Univariable and multivariable Cox regression analyses

\begin{tabular}{lccc}
\hline Univariable analysis & HR & $\mathbf{9 5} \% \mathbf{C I}$ & $\boldsymbol{P}$ value \\
\hline L0 vs L1 & 3.069 & $1.931-4.877$ & .000 \\
V0 vs V1 & 2.018 & $1.188-3.427$ & .009 \\
UICC stage & & & \\
IA & Reference & & .000 \\
IB & 1.208 & $0.740-1.974$ & .450 \\
IIA & 2.723 & $1.449-5.117$ & .002 \\
IIB & 2.265 & $1.224-4.192$ & .009 \\
IIIA & 46.506 & $12.589-171.809$ & .000 \\
Age groups, y & & & \\
$\quad<60$ & Reference & & .077 \\
60-70 & 1.090 & $0.640-1.855$ & .752 \\
>70 & 1.669 & $1.012-2.753$ & .045 \\
Histologic type & & & \\
ACC & Reference & & .665 \\
SCC & 0.827 & $0.534-1.280$ & .394 \\
LCC & 1.669 & $0.550-1.827$ & .993 \\
WHO grade & & & \\
I & Reference & & .302 \\
II & 1.178 & $0.365-3.800$ & .784 \\
III & 1.629 & $0.501-5.302$ & .418 \\
IV & 2.730 & $0.647-11.510$ & .171 \\
Male vs female sex & 1.255 & $0.824-1.914$ & .290 \\
\hline Multivariable analysis & HR & $\mathbf{9 5} \% \mathbf{C I}$ & $\boldsymbol{P}$ value \\
\hline L & & &
\end{tabular}

\section{L category}

L0

$\mathrm{L} 1$

UICC stage

IA

IB

IIA

IIB

IIIA

Age groups, $y$

$<60$

$60-70$

$>70$

$\mathrm{V}$ category

V0

$\mathrm{V} 1$

$H R$, Hazard ratio; $C I$, confidence interval; $L$, lymphatic; $V$, vascular; UICC, Union for International Cancer Control; $A C C$, adenocarcinoma; $S C C$, squamous cell carcinoma; $L C C$, large-cell carcinoma; $W H O$, World Health Organization.

therapy in stage IA NSCLC, ${ }^{21,22}$ available data suggest that vascular invasion should be considered as a criterion in therapy decision making.

The limitations of our study include its retrospective design, the relatively small patient population, and a mixture of histological subtypes. On the other hand, the surgical regimen and type of lymphadenectomy were radical in all patients included in this study. In addition, our data pinpoint toward better risk stratification in early tumor stages. These patients have also not been subject to adjuvant therapy in the past; hence, the potential bias of different adjuvant regimens does not apply to this subgroup.
A larger sample size, with more vascular invasions, would have resulted in clarifying the role of vascular invasion as well.

In conclusion, lymphatic invasion affects overall survival in nonmetastatic completely resected NSCLC patients and represents an independent prognostic factor. In early tumor stages (pT1a and pT1b), it should be considered as a high risk factor and its role in therapy planning and selecting patients in need of adjuvant therapy should be further evaluated. These are especially important because this information is already provided in the routine histopathologic report and no additional assays or molecular profiling is necessary.

We thank Mr Eik Vettorazzi (Department of Medical Biometry and Epidemiology, University Medical Center Hamburg-Eppendorf, Hamburg, Germany) for his help in statistical analysis.

\section{References}

1. Sobin LH, Gospodarowicz M, Wittekind C, eds. TNM Classification of Malignant Tumours. 7th ed. Hoboken, NJ: Wiley-Blackwell; 2010.

2. Chansky K, Sculier JP, Crowley JJ, Giroux D, Van Meerbeeck J, Goldstraw P. The International Association for the Study of Lung Cancer Staging Project: prognostic factors and pathologic TNM stage in surgically managed non-small cell lung cancer. J Thorac Oncol. 2009;4:792-801.

3. Dicken BJ, Graham K, Hamilton SM, Andrews S, Lai R, Listgarten J, et al. Lymphovascular invasion is associated with poor survival in gastric cancer: an application of gene-expression and tissue array techniques. Ann Surg. 2006;243:64-73.

4. Ishii M, Ota M, Saito S, Kinugasa Y, Akamoto S, Ito I. Lymphatic vessel invasion detected by monoclonal antibody D2-40 as a predictor of lymph node metastasis in T1 colorectal cancer. Int J Colorectal Dis. 2009;24:1069-74.

5. Arnaout-Alkarain A, Kahn HJ, Narod SA, Sun PA, Marks AN. Significance of lymph vessel invasion identified by the endothelial lymphatic marker D2-40 in node negative breast cancer. Mod Pathol. 2007;20:183-91.

6. Goeckenjan G, Sitter H, Thomas M, Branscheid D, Flentje M, Griesinger F, et al. Prevention, diagnosis, therapy, and follow-up of lung cancer: interdisciplinary guideline of the German Respiratory Society and the German Cancer Society. Pneumologie. 2011;65:39-59.

7. Naruke T, Suemasu K, Ishikawa S. Lymph node mapping and curability at various levels of metastasis in resected lung cancer. J Thorac Cardiovasc Surg. 1978;76:832-9.

8. Hanahan D, Weinberg RA. Hallmarks of cancer: the next generation. Cell. 2011; 144:646-74.

9. Heon S, Johnson BE. Adjuvant chemotherapy for surgically resected non-small cell lung cancer. J Thorac Cardiovasc Surg. 2012;144:S39-42.

10. Brechot JM, Chevret S, Charpentier MC, Appere de Vecchi C, Capron F, Prudent $\mathrm{J}$, et al. Blood vessel and lymphatic vessel invasion in resected nonsmall cell lung carcinoma: correlation with TNM stage and disease free and overall survival. Cancer. 1996;78:2111-8.

11. Shields TW. Prognostic significance of parenchymal lymphatic vessel and blood vessel invasion in carcinoma of the lung. Surg Gynecol Obstet. 1983;157:185-90.

12. Goldstein NS, Mani A, Chmielewski G, Welsh R, Pursel S. Prognostic factors in T1 NO MO adenocarcinomas and bronchioloalveolar carcinomas of the lung. Am J Clin Pathol. 1999;112:391-402.

13. Hanagiri T, Takenaka M, Oka S, Shigematsu Y, Nagata Y, Shimokawa H, et al. Prognostic significance of lymphovascular invasion for patients with stage I non-small cell lung cancer. Eur Surg Res. 2011;47:211-7.

14. Funai K, Sugimura H, Morita T, Shundo Y, Shimizu K, Shiiya N. Lymphatic vessel invasion is a significant prognostic indicator in stage IA lung adenocarcinoma. Ann Surg Oncol. 2011;18:2968-72.

15. Gabor S, Renner H, Popper H, Anegg U, Sankin O, Matzi V, et al. Invasion of blood vessels as significant prognostic factor in radically resected T1-3N0M0 non-small-cell lung cancer. Eur J Cardiothorac Surg. 2004;25:439-42.

16. Ruffini E, Asioli S, Filosso PL, Buffoni L, Bruna MC, Mossetti C, et al. Significance of the presence of microscopic vascular invasion after complete resection of stage I-II pT1-T2N0 non-small cell lung cancer and its relation with T-size 
categories: did the 2009 7th edition of the TNM staging system miss something? J Thorac Oncol. 2011;6:319-26.

17. Tsuchiya T, Hashizume S, Akamine S, Muraoka M, Honda S, Tsuji K, et al. Upstaging by vessel invasion improves the pathology staging system of non-small cell lung cancer. Chest. 2007;132:170-7.

18. Pechet TT, Carr SR, Collins JE, Cohn HE, Farber JL. Arterial invasion predicts early mortality in stage I non-small cell lung cancer. Ann Thorac Surg. 2004;78:1748-53.

19. Wang J, Chen J, Chen X, Wang B, Li K, Bi J. Blood vessel invasion as a strong independent prognostic indicator in non-small cell lung cancer: a systematic review and meta-analysis. PLoS One. 2011;6:e28844.
20. Tsuchiya T, Akamine S, Muraoka M, Kamohara R, Tsuji K, Urabe S, et al. Stage IA non-small cell lung cancer: vessel invasion is a poor prognostic factor and a new target of adjuvant chemotherapy. Lung Cancer. 2007;56:341-8.

21. Arriagada R, Auperin A, Burdett S, Higgins JP, Johnson DH, Le Chevalier T, et al. Adjuvant chemotherapy, with or without postoperative radiotherapy, in operable non-small-cell lung cancer: two meta-analyses of individual patient data. Lancet. 2010;375:1267-77.

22. Pignon JP, Tribodet H, Scagliotti GV, Douillard JY, Shepherd FA, Stephens RJ, et al. Lung adjuvant cisplatin evaluation: a pooled analysis by the LACE Collaborative Group. J Clin Oncol. 2008;26:3552-9. 